\title{
Swelling Behavior of Bisphenol-A Polycarbonate-Polyoxyethylene Multiblock Copolymers in Ethanol/Water Mixtures
}

\author{
Hideaki TANISUgI, Hiroshi OHNUMA, and Tadao KotaKA* \\ Department of Macromolecular Science, Faculty of Science, \\ Osaka University, Toyonaka, Osaka 560, Japan
}

(Received March 15, 1984)

\begin{abstract}
Swelling behavior was investigated on randomly-coupled $(\mathrm{AB})_{n}$-type multiblock copolymers of bisphenol-A polycarbonate (PC) and polyoxyethylene (POE) in ethanol/water mixtures of various composition. The degree of swelling, $Q_{\mathrm{w}}$, defined as the weight ratio of the swollen and dry films, was dependent not only on PC/POE composition and block length but also on the sample's history. As-cast specimens of the samples having $70 \mathrm{wt} \%$ POE content showed a high maximum in the $Q_{\mathrm{w}}$ vs. solvent composition curve at an ethanol content close to the azeotropic composition. This maximum disappeared by annealing and/or ethanol-conditioning the as-cast films. The swelling behavior of these conditioned films was similar to that of crosslinked POE gels. In the copolymers investigated, hydrophobic PC microdomains may act as physical crosslinks for the hydrophilic POE gel phase. Thus, as-cast specimens may have a large sorptive capacity because of imperfect microphase separation and a resulting loose quasi-network structure.

KEY WORDS Swelling / Water and Ethanol Mixture / Multiblock

Copolymer / Bisphenol-A Polycarbonate-Polyoxyethylene Copolymer /

Microphase Separation / Annealing /
\end{abstract}

Multicomponent polymers or polymeric alloys ${ }^{1-5}$ often undergo microphase separation because of the immiscibility of the constituent polymers. This heterophase structure of polymer alloys has been utilized for designing materials having excellent use-properties and/or special functions, such as thermoplastic elastomers. ${ }^{1-5}$ The unique properties of segmented polyurethanes may also be attributed to a microphase-separated structure consisting of hard and shoft segment domains. ${ }^{1-5}$

To elucidate the relationship between microdomain structure and physical properties of multiblock copolymers, we synthesized a series of randomly-coupled (AB) ${ }_{n}$-type multiblock copolymers of bisphenol-A polycarbonate (PC) and polyoxyethylene (POE) with varying PC/POE content and block length, using the method of Goldberg. ${ }^{6}$ POE is easily crystallizable, rubbery and hydrophilic, while PC is less easily crystallizable, glassy and hydrophobic. We have already investigated the thermal, mechanical and dielectric properties, and water sorption behavior of these copolymers as a function of composition and block length. ${ }^{7-9}$

In this article, we report on the swelling behavior of these copolymers in ethanol/water mixtures of various composition. POE is soluble in both water and ethanol, but not PC. Thus, the swelling behavior of these copolymers should depend strongly on PC/POE composition, block length and solvent composition.

\section{EXPERIMENTAL}

\section{Materials}

The characteristics of the six copolymer samples $^{8}$ used in this study are summarized in

\footnotetext{
* To whom correspondence should be addressed.
} 
Table I. Characteristics of multiblock copolymers $^{\mathrm{a}}$

\begin{tabular}{|c|c|c|c|c|c|}
\hline \multirow{2}{*}{$\begin{array}{l}\text { Sample code } \\
\text { (BXEY) }\end{array}$} & POE Content & \multirow{2}{*}{$X$} & \multirow{2}{*}{$Y$} & \multirow{2}{*}{$Z$} & \multirow{2}{*}{$n$} \\
\hline & $\mathrm{wt} \%$ & & & & \\
\hline B22E46 & 26.9 & 22.9 & 46 & 1.1 & 12 \\
\hline B9E14 & 22.8 & 9.6 & 14 & 1.1 & 21 \\
\hline B13E68 & 48.2 & 13.9 & 68 & 1.1 & 8 \\
\hline B5E23 & 47.7 & 5.5 & 23 & 1.2 & 35 \\
\hline B5E68 & 70.0 & 6.1 & 68 & 1.2 & 11 \\
\hline B2E23 & 70.0 & 2.8 & 23 & 1.6 & 32 \\
\hline
\end{tabular}

Table I. The samples are coded as BXEY, the symbols $\mathrm{X}$ and $\mathrm{Y}$ denoting the average degree of polymerization of $\mathrm{PC}$ blocks and POE precursors, respectively. The values of $\mathrm{X}$ and average number $Z$ of coupled POE precursors in a single POE block were calculated from the feed-product composition under the assumption that coupling of the bisphenol-A and POE precursors occurs randomly. The details for this are described elsewhere. ${ }^{7-9}$

An impurity-free PC homopolymer sample (Teijin Co., Ltd., K-1300) used as a reference had a number-average molecular weight, $M_{n}$, of 29,800. A crosslinked POE film also used as a reference was prepared as follows. ${ }^{10} \mathrm{~A}$ commercial POE prepolymer (Nakarai Chemicals Ltd.; nominal molecular weight, 1850-2150) was mixed with $17 \mathrm{~mol} \%$ trimethylolpropane, $50 \mathrm{~mol} \%$ 4,4'-diphenylmethane diisocyanate and $0.05 \mathrm{wt} \%$ catalyst, di- $n$-butyltin dilaurate, under the $\mathrm{N}_{2}$ stream. After being kept at $100^{\circ} \mathrm{C}$ for $10 \mathrm{~min}$ in a molding cell, the mixture was hot-pressed at $20 \mathrm{~kg} \mathrm{~cm}^{-2}$ in situ for $1 \mathrm{~h}$. The compression molded film was left standing at least for one week before use, because the crosslinking reaction proceeded very slowly.

Films of the copolymers and PC homopolymer were cast from $2 \mathrm{wt} \%$ chloroform solutions at room temperature by evaporating the solvent slowly for 2 to 3 days. They were then dried under a vacuum of $10^{-2}$ torr to constant weight. Whenever necessary, the films were further annealed at $60^{\circ} \mathrm{C}$ for $24 \mathrm{~h}$.

\section{Methods}

For swelling measurements, thoroughly dried films were cut into pieces about $10 \times 30 \times$ $0.1 \mathrm{~mm}^{3}$ (approximately $30 \mathrm{mg}$ ), and soaked in ethanol/water mixtures of varied composition thermostated (within $\pm 0.1^{\circ} \mathrm{C}$ ) at desired temperatures. The swollen films were weighed by a Mettlar semi-micro balance (within $\pm 0.1 \mathrm{mg}$ ) every $24 \mathrm{~h}$ until a constant weight was reached. The maximum degree of swelling, $Q_{\mathrm{w}}$, was calculated as the ratio of the weights of the swollen and dry films. It usually took 4 days to reach swelling equilibrium.

Dynamic mechanical measurements were made on a Rheovibron DDV-II (ToyoBaldwin Co.) at $110 \mathrm{~Hz}$ in a temperature range from 150 to $450 \mathrm{~K}$ at a heating rate of 1 $\mathrm{K} \mathrm{min}^{-1}$. Differential scanning calorimetry (DSC) was carried out on a Rigaku Denki Model 8055 DSC apparatus in a range from 170 to $570 \mathrm{~K}$ at a heating rate of $10 \mathrm{~K} \mathrm{~min}^{-1}$.

\section{RESULTS}

\section{PC/POE Composition and Block Length Dependence}

Figures 1 and 2 show the degree of swelling $Q_{\mathrm{w}}$ vs. solvent composition curves at $25^{\circ} \mathrm{C}$ for BXEY polymers with long and short blocks, respectively. For comparison, Figure 1 also shows the $Q_{\mathrm{w}}$ of crosslinked POE and PC homopolymers. The $Q_{\mathrm{w}}$ of crosslinked POE gradually decreased with increasing ethanol content up to about $85 \mathrm{vol}^{\circ} \%$, and, then, rapidly decreased to the value in pure ethanol. The PC sample hardly swelled at any mixture composition. However, the $Q_{\mathrm{w}}$ for the BXEY copolymers first increased to a maximum at around 80 to $90 \%$ ethanol content and then decreased sharply. This tendency was more pronounced for copolymers of higher $(70 \mathrm{wt} \%)$ POE content. As shown in Figure 1, B5E68 


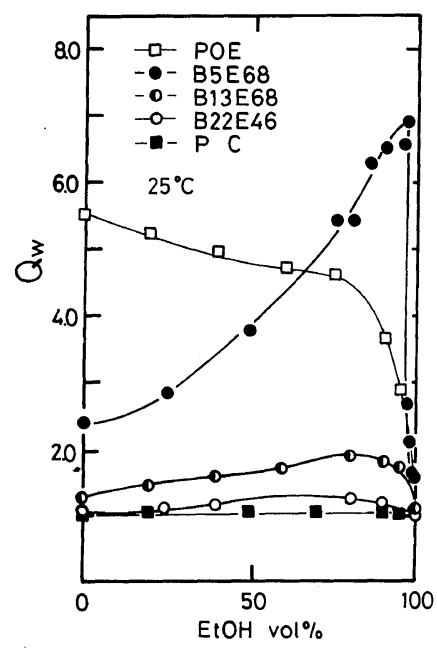

Figure 1. Plot of $Q_{\mathrm{w}} v$ s. solvent composition at $25^{\circ} \mathrm{C}$ for homopolymers and as-cast BXEY polymers of long blocks and varying $\mathrm{PC} / \mathrm{POE}$ ratio.

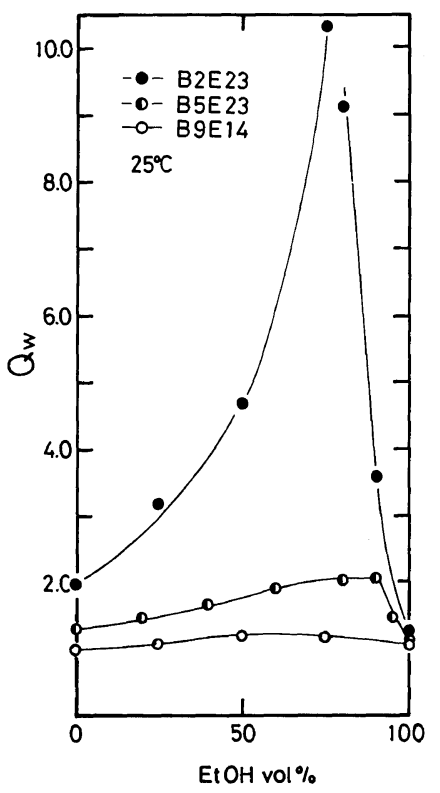

Figure 2. Plot of $Q_{\mathrm{w}} v s$. solvent composition at $25^{\circ} \mathrm{C}$ for as-cast BXEY polymers of short blocks and varying $\mathrm{PC} / \mathrm{POE}$ ratio.

exhibited a sharp $Q_{\mathrm{w}}$ maximum for ethanol/ water mixtures near. the azeotropic composition (96 vol\% ethanol). Figure 2 shows that B2E23 having the same POE content as B5E68 but short blocks becomes soluble in mixtures of 85 to $90 \mathrm{vol} \%$ ethanol, i.e., $Q_{w}$ becomes infinite. Thus, we found the swelling behavior of the as-cast specimens of high POE content to be very different from that of crosslinked POE.

When the swelling data for $70 \%$ POE containing samples, B5E68 and B2E23, are compared with those for $50 \%$ POE containing samples, B13E68 and B5E23, respectively, we notice that $Q_{\mathrm{w}}$ for the former two samples are always larger than those for the latter two. Incidentally, the two samples in each pair were the same in the length of POE blocks but different in the length of PC blocks. However, the difference in their swelling behavior was much larger than that expected from the additivity of the contributions of the two components.

\section{Temperature Dependence}

Figure 3 shows the $Q_{\mathrm{w}}$ vs. solvent composition curves for as-cast B5E68 at four different temperatures. It can be seen that with an increase in temperature, $Q_{w}$ increases and the cosolvent composition at the $Q_{\mathrm{w}}$ peak shifts to a region of higher ethanol content. At $35^{\circ} \mathrm{C}$ and above, the as-cast B5E68 specimen becomes soluble in the mixtures of high ethanol content. Graham et al. ${ }^{11}$ reported that $Q_{\text {w }}$ for crosslinked POE hydrogels in water decreases with increasing temperature. Our BXEY polymers exhibited a tendency opposite to Graham's crosslinked POE hydrogels.

\section{Effect of Thermal Treatments}

Since thermal treatment was found ${ }^{8}$ to affect the morphology and properties of the multiblock copolymers in this study, we compared the swelling behavior of their as-cast and annealed (at $60^{\circ} \mathrm{C}$ for $24 \mathrm{~h}$ ) specimens. Figures 4 and 5 show the $Q_{\mathrm{w}} v s$. solvent composition curves for as-cast and annealed specimens of B13E68 and B5E23 with 50\% POE content and those of B5E68 and B2E23 with 70\% POE content, respectively. It can be seen that the $Q_{\mathrm{w}}$ values of the annealed specimens are con- 


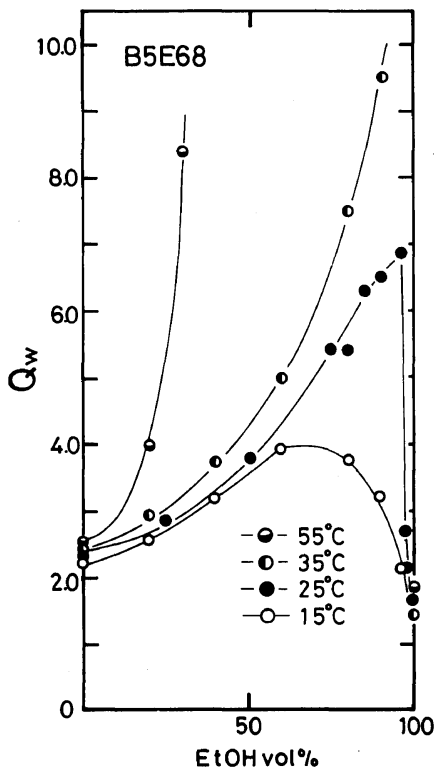

Figure 3. Plot of $Q_{\mathrm{w}} v s$. solvent composition for as-cast B5E68 specimens at different temperatures.

sistently lower than those of the as-cast specimens and that the $Q_{\mathrm{w}}$ maximum shifts to a lower ethanol content with annealing. On the other hand, no annealing effect was found for $Q_{\mathrm{w}}$ of B22E46 and B9E14 having low $(25 \mathrm{wt} \%)$ POE content.

Figure 6 shows the temperature dependence of the storage $\left(E^{\prime}\right)$ and loss $\left(E^{\prime \prime}\right)$ moduli and $\tan \delta$ for the as-cast and annealed specimens of B5E68, B2E23 and B13E68. For the annealed specimens of these samples, a sharp decrease in $E^{\prime}$ reflecting the glass transition of the POE rich phase occurs at a temperature lower than that for the corresponding as-cast specimens. Also, the annealed specimens show plateaus extending to high temperatures. The $E^{\prime} v s$. temperature curves for the annealed specimens of B2E23 and B13E68 exhibit a small but significant peak around $400 \mathrm{~K}$ and in the 420 to $470 \mathrm{~K}$ region, respectively. According to our previous studies $^{7-9}$ on the viscoelastic and thermal behavior of BXEY copolymers, these peaks may be attributed to the recrystallization of PC blocks. ${ }^{8}$ In Figure 6, an anom-

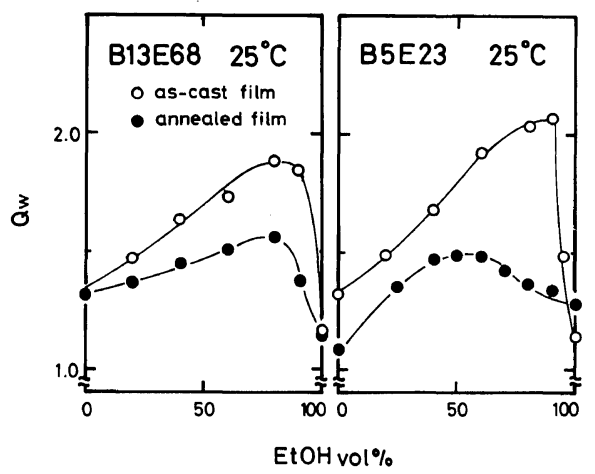

Figure 4. $Q_{\mathrm{w}} v s$. solvent composition at $25^{\circ} \mathrm{C}$ for ascast and annealed B13E68 (left) and B5E23 (right) specimens of $50 \%$ POE content.

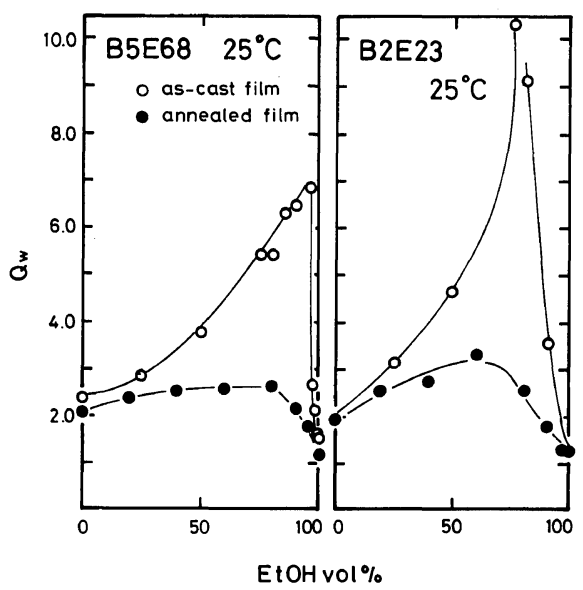

Figure 5. $Q_{\mathrm{w}} v s$. solvent composition at $25^{\circ} \mathrm{C}$ for ascast and annealed B5E68 (left) and B2E23 (right) specimens of $70 \%$ POE content.

alous peak is seen in the $E^{\prime} v s$. temperature curve for B2E23 in the range from about 270 to $300 \mathrm{~K}$. This anomally corresponds to the initiation of crystallization and melting in POE domains, as discussed in our previous publication. $^{8}$

\section{Effect of Conditioning by Pure Solvents}

Figure 7 compares the $Q_{\mathrm{w}} v s$. solvent composition curves for B5E68 specimens conditioned at $25^{\circ} \mathrm{C}$ in pure water and in pure ethanol. The values of $Q_{\mathrm{w}}$ for the former was determined by successively changing the 


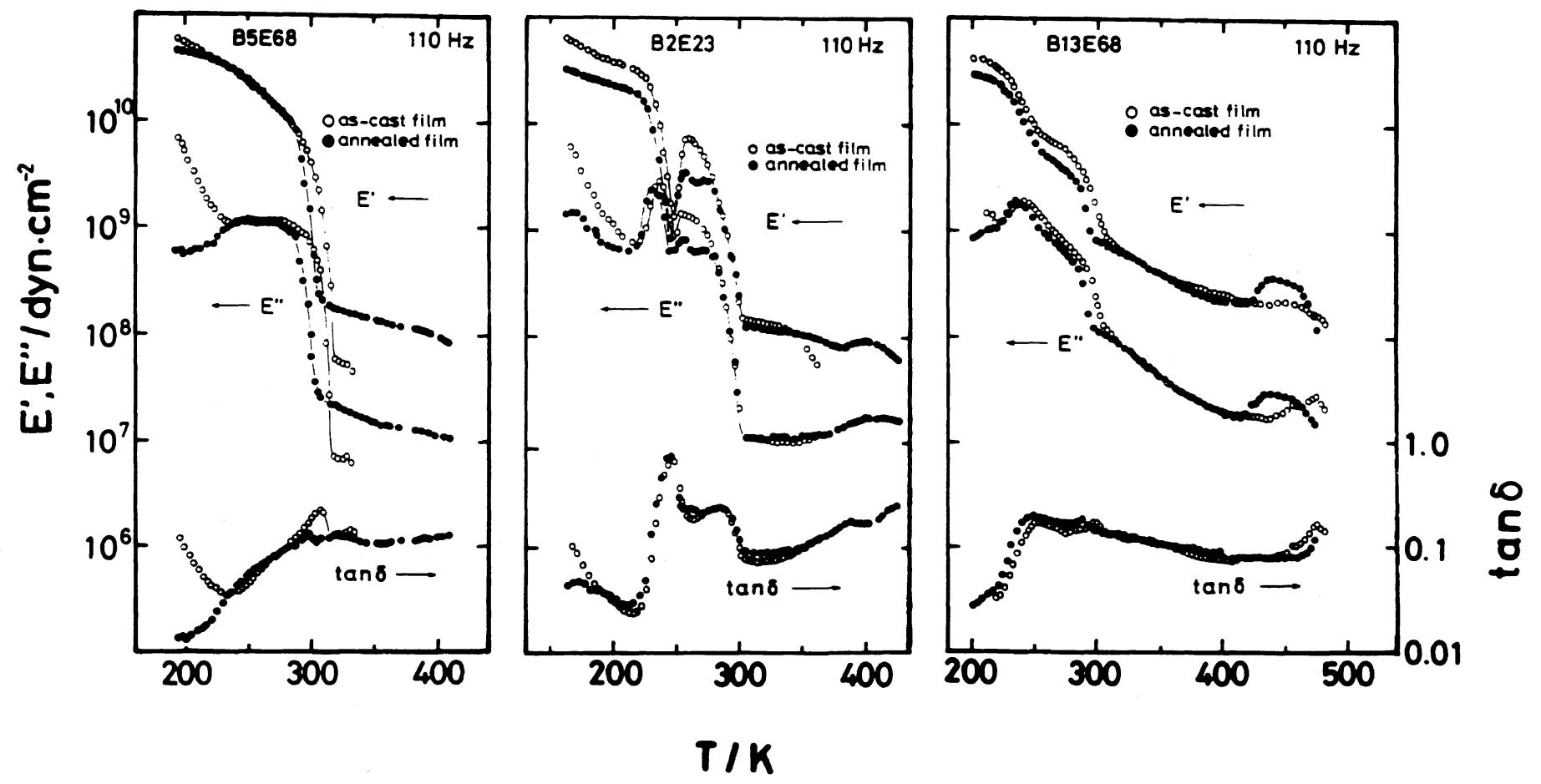

Figure 6. Effect of annealing on temperature dependence of $E^{\prime}, E^{\prime \prime}$ and $\tan \delta$ of (a) B5E68, (b) B2E23, and (c) B13E68 specimens. 


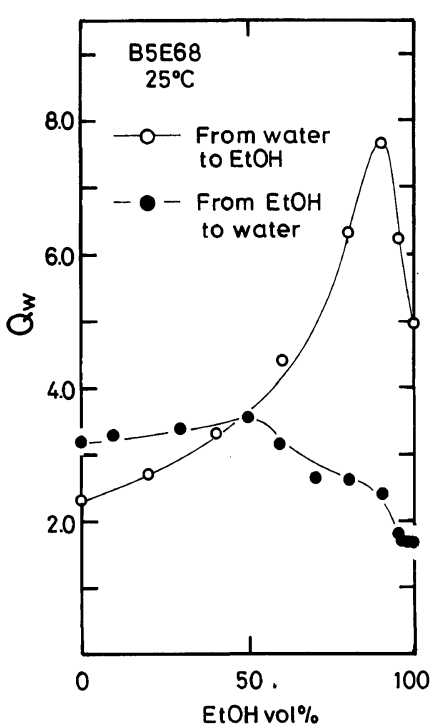

Figure 7. $Q_{\mathrm{w}} v s$. solvent composition for B5E68 films conditioned at $25^{\circ} \mathrm{C}$ in pure water and ethanol. ethanol content from pure water to ethanol, and those for the latter by the other way around. We see a striking change in the $Q_{\mathrm{w}} v s$. solvent composition curve for the ethanolswollen specimen, which resembles that of the annealed specimen shown in Figure 5.

Figure 8 compares the DSC thermograms of the as-cast and annealed specimens of B5E68, B2E23, and B13E68. For B5E68 the thermograms of an ethanol conditioned specimen are also shown. The as-cast specimens show an endothermic peak at about $506 \mathrm{~K}$, while the annealed specimens show an exothermic peak in the range from 450 to $590 \mathrm{~K}$. According to our previous studies, ${ }^{7-9}$ the former peaks can be assigned to melting of PC crystallites, and the latter to recrystallization of PC blocks. No melting peak of PC crystallites was detected

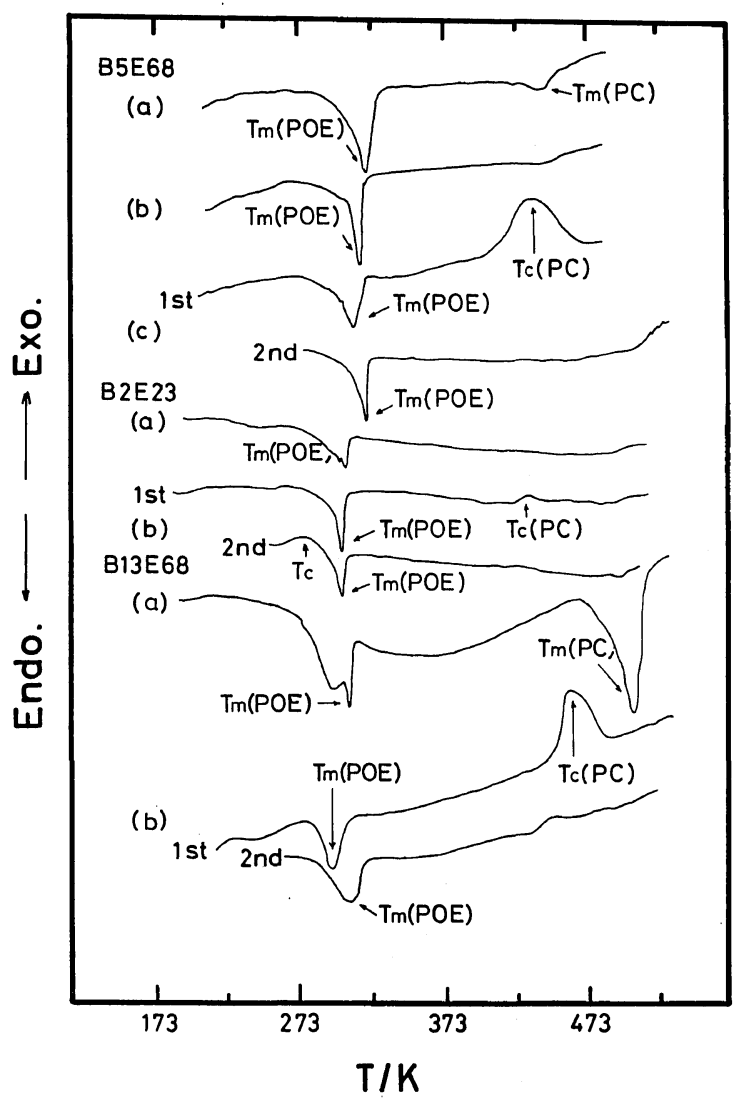

Figure 8. DSC thermograms of (a) as-cast and (b) annealed (at $60^{\circ} \mathrm{C}$ for $24 \mathrm{~h}$ ) specimens of B5E68, B2E23, and B13E68. For B5E68, those of (c), an EtOH conditioned specimen, are also shown. 
for the annealed specimens since thermal decomposition of PEO blocks began below the melting temperature. The thermograms for an ethanol-conditioned specimen of B5E68 also exhibited a fairly large exothermic peak ascribable to $\mathrm{PC}$ recrystallization.

In each measurement, we lowered the temperature to $0^{\circ} \mathrm{C}$ just after passing through the exothermic peak, and reheated for a second measurement. None of the second-run chromatograms showed an exothermic peak in the same temperature range, indicating that the PC phase had been completely crystallized during the first heating process.

\section{DISCUSSION}

The BXEY copolymers examined here are typical short-segment multiblock copolymers composed of crystallizable segments. Obviously, their swelling behavior should be governed not only by solvent-polymer interactions but as well by microdomain structures in the specimens. Both water and ethanol are good solvents for POE blocks, but nonsolvents for PC blocks which swell slightly in the latter. Since both PC and POE segments are crystallizable, microphase separation in the BXEY polymers is influenced not only by the thermodynamic immiscibility of the components but also by the crystallizability of each component. ${ }^{12}$ Nevertheless, in such shortsegment multiblock copolymers, microphase separation occurs less completely, and the copolymers presumably have more diffuse and mixed interphases than usual AB diblock copolymers. $^{7,8}$ These structural characteristics also influence swelling behavior in ethanol/ water mixtures.

In our previous study on water sorption by the BXEY polymers at low relative humidities, $\mathrm{RH},{ }^{9}$ it was found that water molecules diffuse into and are absorbed essentially by amorphous POE domains. Therefore, the sorption capacity of the copolymers at low RH could be related to the amorphous POE content.
However, in BXEY specimens highly swollen in ethanol/water mixtures, POE crystallites are dissolved in the mixtures and only the PC domains act as physical crosslinks. Particularly, in B2E23 and B5E68 having high POE content and shorter PC blocks, PC blocks work less effectively as crosslinks than those in B5E23 and B13E68 having lower POE content and longer PC blocks. The former two may have a rather loose network structure and, hence, can take up a larger quantity of cosolvent than the latter two. This partly explains why as-cast specimens, especially those of B2E23 and B5E68, swell in mixtures of high ethanol content.

It is known that some polymers dissolve in a cosolvent over a limited range of composition. ${ }^{13}$ In BXEY polymers, PC microdomains acting as physical crosslinks become swollen, softened and eventually dissolve with rise in temperature. This should weaken the tension acting on POE strands and, hence, promote specimen swelling and may account for the fact that in mixtures of high ethanol content, some BXEY copolymers swell much more than crosslinked POE and become soluble under certain conditions.

Thermal annealing obviously promotes microphase separation, and hence influences swelling behavior. As evident from Figures 4 and 5 , the most pronounced difference in swelling behavior between the as-cast and annealed specimens of BXEY copolymers was observed for POE rich samples, especially B2E23. The as-cast film of B2E23 was soluble in mixtures of 85 to $90 \%$ ethanol content, but when annealed, became insoluble at any composition. The swelling behavior of the annealed films was thus qualitatively similar to that of crosslinked POE.

Since our samples were annealed above the melting temperature of POE crystallites and below the glass transition temperature of PC blocks, further microphase separation first occurred mainly in the POE-rich domains. Thus, the pure POE phase was segregated from the 
mixed interphase, and the remaining PC rich phase tended to crystallize, giving more effective crosslinks for the POE blocks. This structural change induced by annealing is reflected in the dynamic mechanical behavior shown in Figure 6. A similar effect was also observed in the DSC thermograms, as shown in Figure 8.

In conditioning with ethanol, ethanol probably acts as a plasticizer in the PC-rich phase and promotes microphase separation. In fact, a large PC crystallization peak was observed in the thermograms of ethanol-conditioned specimens, and the sorption capacity of the films decreased significantly by this conditioning.

All these findings suggest that annealing and/or ethanol conditioning promote microphase separation of $\mathrm{POE}$ and $\mathrm{PC}$ in the intermixing phase so that the $\mathrm{PC}$ phase become more effective crosslinks for the POE blocks. Such a change in the microphase separated structures explains why the swelling behavior of annealed and/or ethanol conditioned specimens became similar to that of crosslinked POE.

Acknowledgements. We wish to thank Dr. Toshitake Suzuki for his valuable discussion and advice in carrying out the experiments. The PC homopolymer sample was a gift from Dr. Yoji Murakami, Teijin Co., whom we also wish to thank. This work was supported by the Ministry of Education, Science and Culture (Monbusho) under grants 543026 (to TK) and
58350053 (Professor Michio Kurata, Kyoto University, the coordinator), which are gratefully acknowledged.

\section{REFERENCES}

1. A. Noshay and J. E. McGrath, "Block Copolymers-Overviews and Critical Survey," Academic Press, New York, N. Y., 1977.

2. S. A. Manson and L. H. Spering, "Polymer Blend and Composite," Plenum Press, New York, N. Y., 1977.

3. S. L. Cooper and G. M. Estes, Ed, "Multiphase Polymers," Adv. Chem. Series 176, The American Chemical Society, Washington D.C., 1979.

4. T. Kotaka and M. Shen, Ed, "Proceedings of USJapan Joint Seminar on Multicomponent Polymers," Kyoto, 1978; J. Macromol. Sci., Phys., B17, 2 (1980).

5. T. Kotaka, F. Ide, K. Ogino, and T. Nishi, Ed, "Porima Aroi (Polymer Alloys)," Tokyo Kagakudojin, Tokyo, 1981.

6. E. P. Goldberg, J. Polym. Sci., C, 4, 707 (1964).

7. T. Suzuki and T. Kotaka, Macromolecules, 13, 1495 (1980).

8. T. Suzuki and T. Kotaka, Polym. J., 15, 15 (1983).

9. T. Suzuki, H. Chihara, and T. Kotaka, Polym. J., 16, 129 (1984).

10. H. Adachi, S. Nishi, and T. Kotaka, Polym. J., 14, 985 (1982).

11. N. B. Graham, N. E. Nwachuku, and D. J. Walsh, Polymer, 23, 1345 (1982).

12. S. Krause, "Colloidal and Morphological Behavior of Block and Graft Copolymers," G. Molau, Ed, Plenum Press, New York, N. Y., 1971, pp223-234; "Block and Graft Copolymers," J. J. Burke and V. Weiss, Ed, Syracuse Univ. Press, Ithaca, New York, N. Y., 1973, pp 143-155.

13. See, for example, H. Morawetz, "Macromolecules in Solution," Interscience Publishers, New York, N. Y., 1965, Chapter II. 